\title{
Satisfaction with an In-House Nasal Foreign Body Removal Manikin: A Randomized Controlled Trial
}

\author{
Pittayapon Pitathawatchai ${ }^{1}$ \\ Sittichoke Anuntaseree ${ }^{2}$ \\ Varah Yuenyongviwat ${ }^{2}$ \\ 'Department of Otolaryngology Head \& \\ Neck Surgery, Faculty of Medicine, Prince \\ of Songkla University, Hatyai, Thailand; \\ ${ }^{2}$ Department of Orthopedics, Faculty of \\ Medicine, Prince of Songkla University, \\ Hatyai, Thailand
}

\begin{abstract}
Introduction: Nasal foreign bodies can lead to life-threatening conditions. Hence, it is a necessity that physicians be well trained in the appropriate procedures for removal of nasal foreign bodies. However, training on real patients is not only unpractical it is also too dangerous, due to risk of foreign body aspiration during the procedure. Therefore, our goal was to construct a manikin, with a specific design, to serve all possible needs for training. Methods: We developed an in-house manikin from 2 materials; these being flexible polyurethane foam and silicone. Silicone, which has elasticity similar to nasal alae, was used to develop the detachable nose and nasal cavity, whilst polyurethane foam, which is light and easy to carry, was used to develop the head. The in-house manikin was compared with a commercial manikin for satisfaction after a nasal foreign body removal procedure was performed in both groups, by 37 physicians, after conducting a randomized controlled trial with a crossover design.
\end{abstract}

Results: The satisfaction scores of the in-house manikin were statistically significantly higher than the satisfaction scores of the commercial manikin for 6 dimensions: proper size, ease of use, ease of maintenance, flexibility of nasal alae relative to actual anatomy, similarity of the nasal cavity relative to actual anatomy and confidence that the manikin can upskill the medical students ability to practice on real patients ( $\mathrm{p}$ value $<0.05$ ).

Conclusion: This in-house nasal foreign body removal manikin design had high satisfaction for training and could be used to develop further nasal foreign body removal manikins in the future. Keywords: manikin, nasal foreign body removal

\section{Introduction}

Foreign bodies in the ear, nose and throat (ENT) accounted for approximately $10 \%$ of emergency ENT conditions observed at an emergency department. ${ }^{1}$ Among all foreign bodies in ENT, the nasal cavity is a common structure in which up to nearly $50 \%$ of ENT foreign bodies could be found. ${ }^{2,3}$ The most common location, where nasal foreign bodies lodge, is between the inferior turbinate and nasal floor. ${ }^{4}$ Generally, most patients diagnosed with nasal foreign bodies are children under 5 years. ${ }^{5}$ The reasons that foreign bodies are commonly found in this age group may be related to the fact that they tend to be inquisitive about their surroundings as well as being more autonomous to explore their environments. ${ }^{6}$ The typical presentations of nasal foreign bodies are sneezing and unilateral serous rhinorrhea at the initial stage, with the progression of unilateral purulent nasal discharge for a few days. ${ }^{7}$

Although, some complications, including rhinosinusitis, epistaxis, lacerations and rhinolith formation, could be observed without serious consequences, ${ }^{8}$ a lifethreatening condition, such as foreign body aspiration, can occur when the foreign
Correspondence: Varah Yuenyongviwat Department of Orthopedics, Faculty of Medicine, Prince of Songkla University, 15 Karnjanavanich Road, Hat Yai, Songkhla, 90II0, Thailand

Email varahortho@gmail.com 
body is displaced from the nasal cavity into the respiratory tract: resulting in obstruction that compromises the airway. Therefore, it is crucial that physicians are capable to diagnose as well as treat nasal foreign bodies without causing unnecessary complications. However, minor complications; for instance, lacerations and pain, might develop at any time while the foreign body is being removed. ${ }^{9}$ Also, an improper technique for foreign body removal from the nasal cavity can dislodge the foreign body from the nose, but completely obstruct the airway, leading to a life-threatening condition; which should be always kept in mind. ${ }^{10}$ It has been reported that these complications are usually related to repeated attempts during nasal foreign body removal. ${ }^{5}$ As a result, it is a necessity that physicians are well trained in appropriate procedures for removal of nasal foreign bodies safely and successfully, with minimal attempts.

To have practical skills for nasal foreign body removal, adequate training is required; even though two barriers are encountered. Firstly, as the success rate in the removal is negatively correlated to the number of attempts, ${ }^{11}$ the physician having the most experience is prioritized to maximize the success rate; in addition to minimizing any life-threatening condition. Consequently, physicians with less experience tend to be the least desirable option to perform such procedures. Additionally, it is highly unlikely in said event that any trainers are on hand to take charge. Secondly, nasal foreign bodies are generally considered as ENT emergencies; thus, it is not practical to provide real cases under supervision for trainers during specific training periods. Although training by placing the foreign body into a healthy individual's nasal cavity as a simulated patient is possible, no one can guarantee that a serious complication; such as, foreign body aspiration, would not occur in the simulated patient. Therefore, this practice would be considered both dangerous and possibly unethical. To overcome any issues regarding training in real persons, using a simulated manikin, instead of an actual or simulated patient, is another option. This also takes into consideration that the training is able to be held at any time period, without any issues in concerns to minor or serious complications. In fact, it has been shown that simulated manikins are a useful alternative to a simulated patient, providing a statistically significant increase in students' knowledge and confidence prior to exposure to real patients. ${ }^{12}$ However, it should be noted that commercial manikins currently available in the marketplace are somewhat expensive, and do not always serve all of the actual needs of end users. For instance, in terms of a nasal foreign body removal manikin, the softness or structures of a manikin might not be similar to actual human anatomy. This is depending on both how well it is designed and the quality of materials used, which can also substantially increase the cost. Additionally, in foreign body removal procedures, the manikin's nasal alae; where the nasal speculum must be placed to widen the nasal cavity entrance, can be easily torn due to multiple attempts of removal during training activities. Once this has occurred, in order to continue using the simulated manikin, the torn alar needs immediate repair; by the manufacturer, otherwise the manikin becomes unsuitable for use in foreign body removal procedures. Because of this, not only do the repairs incur added cost, the time spent during maintenance can effect training schedules. Thus, not only using the manikin becomes costly, but also maintenance downtimes are sometimes an issue to serve the needs of end users.

Therefore, the aim of this present study was to devise a low-cost manikin, with a specific design to serve all possible needs of end users, while still keeping its function as necessary for training.

\section{Methods}

\section{Manikin Development}

The in-house manikin prototype was developed with 2 materials; these being flexible polyurethane (PU) foam and silicone. PU foam, which is light and able to be carried with ease, was initially created by mixing polyether polyol and isocyanate together, and was used to construct the head. The silicone, which has elasticity similar to the nasal alae, was used to develop a detachable nose and nasal cavity. This in-house manikin was specially designed as having a detachable nose and nasal cavity, which can be promptly replaced with spare parts in case it requires immediate maintenance; for example, when the nasal alar is accidentally torn after multiple attempts during training. Moreover, the detachable nose and nasal cavity can be removed as one piece in order to allow users to completely check where the foreign body is placed, or to explain the anatomy of the nose and nasal cavities during training, so as to enhance useful feedback to trainees (Figure 1A). The size of the manikins head was $21 \times 16 \times 18$ centimeters (height $\times$ width $\times$ depth), with 

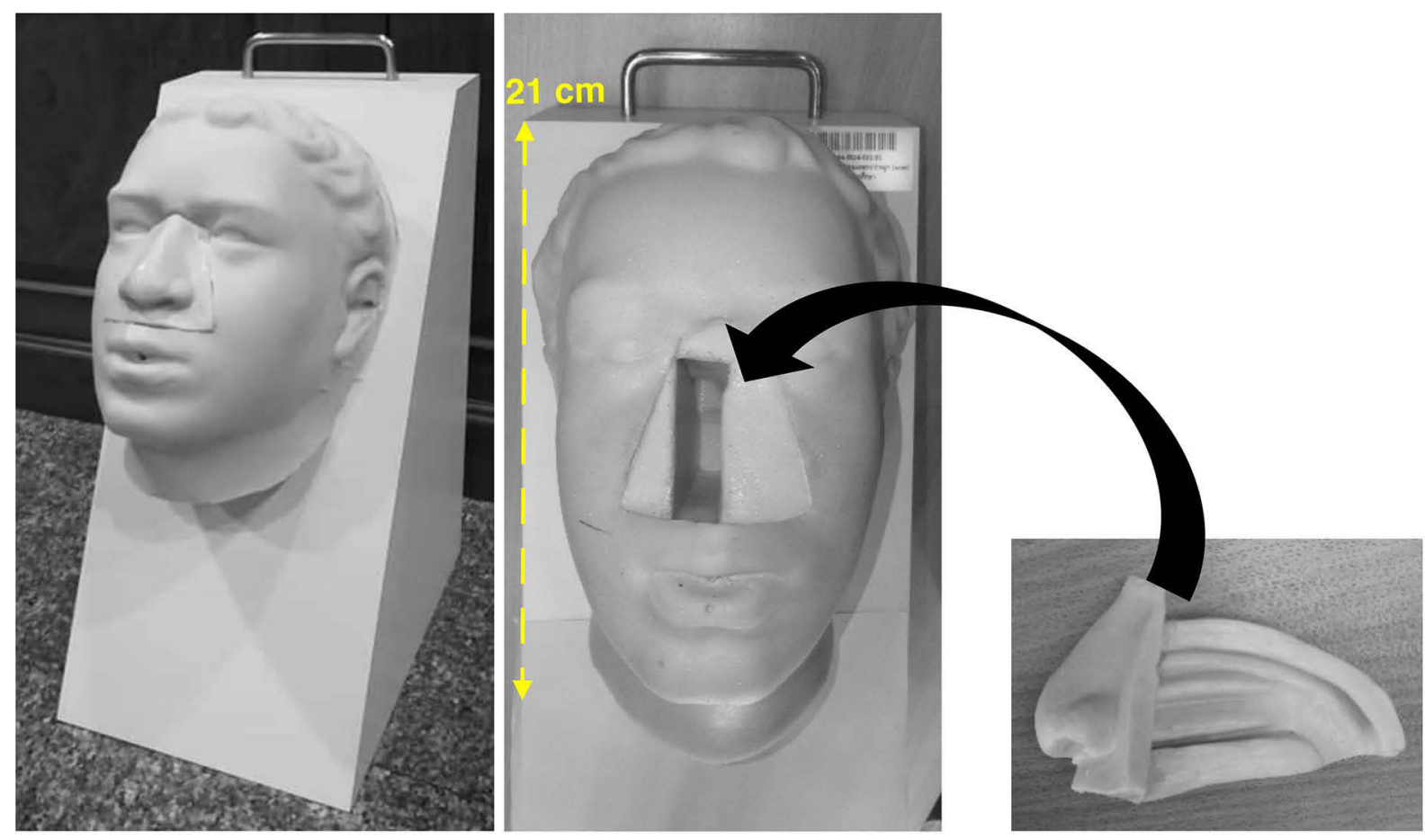

A
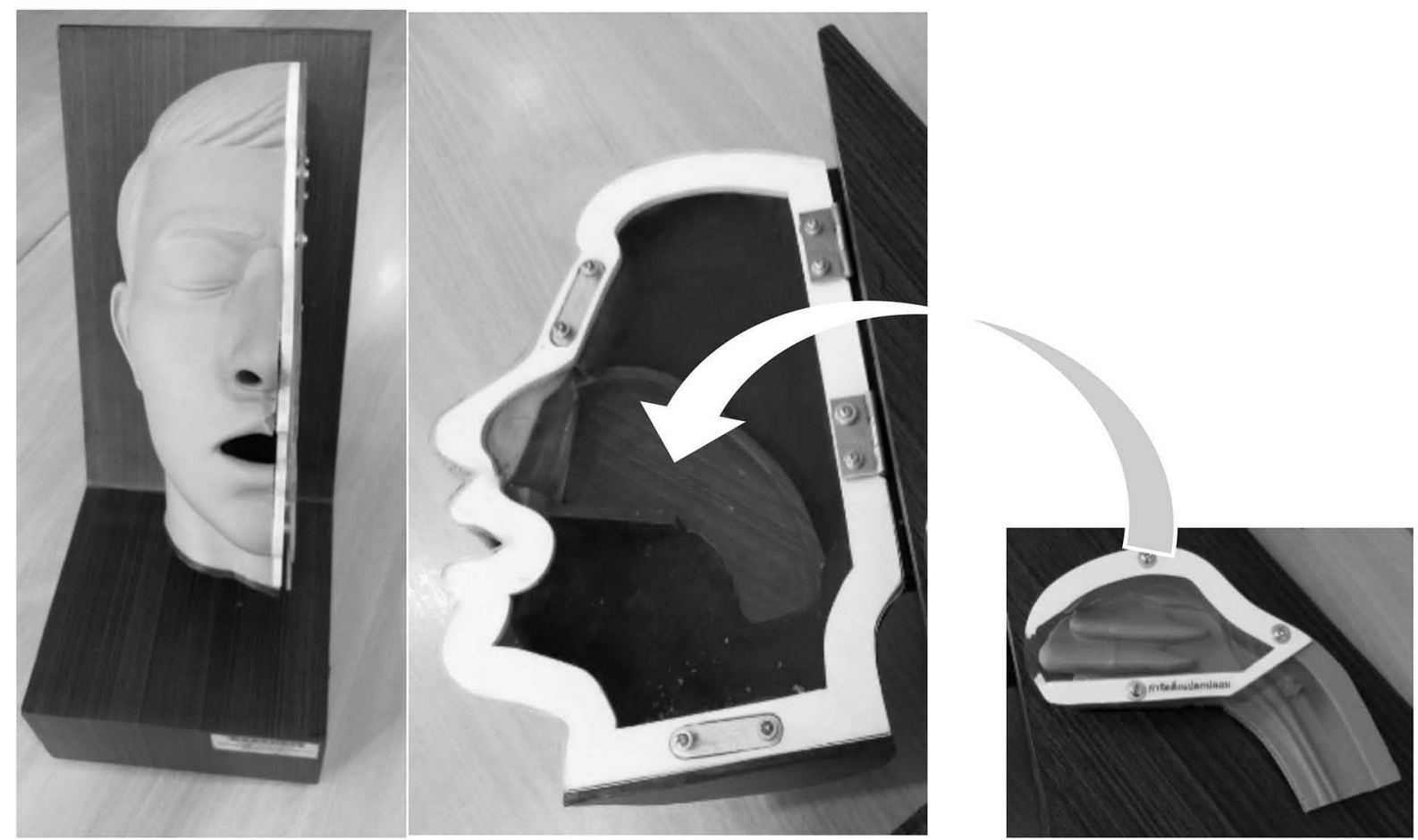

B

Figure I The in-house manikin (A) and the commercial manikin (B).

its total size, including the base, being $42 \times 20 \times 27.5$ centimeters (height $\times$ width $\mathrm{x}$ depth).

The current commercial manikin (E001, BRILLIANT RUBBER CO, LTD, Songkhla, Thailand) has a different design from our in-house manikin. Specifically, this commercial manikin's head was manufactured with a half head, showing the nasal cavity from a medial view. In addition, only the nasal cavity section can be removed from the lateral side of this commercial manikin, while the nose is fixed with the head and cannot be detached (Figure 1B). 


\section{Nasal Foreign Body Removal Test}

A randomized controlled trial, with a crossover design was performed. Thirty-seven participants, including ENT residents, ENT fellows and ENT surgeons, who had experience in nasal foreign body removal were included. The study adhered to the tenets of the Declaration of Helsinki, in accordance with the principles of Good Clinical Practice and the Consolidated Standards of Reporting Trials Statement (CONSORT) (Figure 2). This trial was registered at the Clinical Trials.gov (NCT04901026). The study was approved by the institutional review board.
Participants were allocated into 2 groups, via a computer-generated randomization in blocks of four, and a sealed envelope system. The first group of 19 participants performed the nasal foreign body removal procedure using the in-house manikin. Later, they performed exactly the same procedure using the aforementioned commercial manikin. The second group of 18 performed the same procedure of nasal foreign body removal using the same commercial manikin first; after which, they performed the same procedure, using the identical steps, with the in-house manikin. All participants were instructed to use only a headlight, a nasal speculum and a right-angle

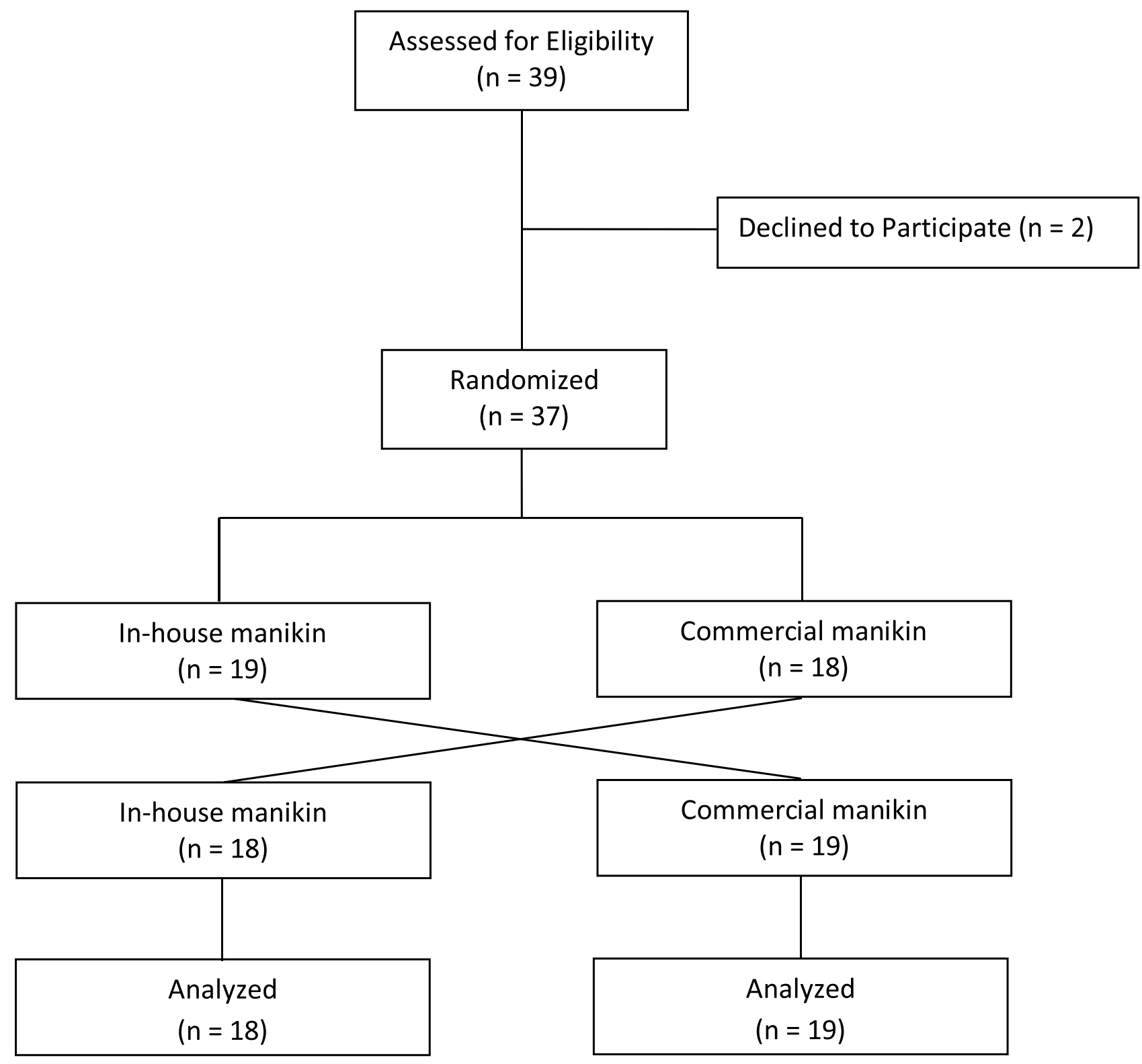

Figure 2 Flow diagram. 
hook to remove a red bead, which was placed at the right nasal floor between the inferior turbinate and the septum of both the in-house and commercial manikins.

A questionnaire regarding the satisfaction in using both manikins, with a 5-point Likert scale (1-very unsatisfied to 5-very satisfied), was used to interview all participants promptly after the procedures were finished. The questionnaire included 9 dimensions, consisting of: proper size, proper weight, ease of use (the ease in which a foreign body could be inserted), ease of cleaning, ease of maintenance, flexibility of the nasal alae relative to actual anatomy, similarity of the nasal cavity relative to actual anatomy, whether it was well designed for teaching, and confidence that the manikin can upskill medical students ability to practice on a real patient. All of these dimensions were explained by the first author before the participants performed the procedure to ensure that they understood and responded to the questions correctly; additionally, any queries raised before, during or after the procedure were instantly answered by the first author.

\section{Statistical Analysis}

A Wilcoxon signed-rank test was used to compare the satisfaction scores between the in-house and commercial manikin. A P value lower than 0.05 was considered as statistically significant.

Simple and multiple linear regression were used to assess which variables were independently associated, with a total satisfaction score difference between the inhouse and commercial manikins. Baseline characteristics, including medical specialty, age, gender, time experience as a general physician or an ENT specialist, were assigned as independent variables. The total satisfaction score difference between the in-house and commercial manikins was assigned as a dependent variable. This total satisfaction score difference was calculated from a different value of score summations in each dimension between the inhouse and commercial manikins. The Shapiro-Wilk test was used to test the normality of the dependent variables. In the simple regression analysis, a threshold of a $\mathrm{p}$ value less than 0.1 was used to identify candidates for inclusion in the multiple linear regression.

The R software version 3.1.0 (R Foundation, Vienna, Austria) was used for the statistical analysis.

\section{Results}

Finally, there were 15 ENT surgeons, 2 ENT fellows and 20 ENT residents, aged between 24 and 57 years included
Table I Demographic Data of Study Participants $(\mathrm{N}=37)$

\begin{tabular}{|l|c|}
\hline Baseline characteristic & \\
\hline Age in years (median) & 30 \\
\hline Gender & $\mathrm{N}(\%)$ \\
Female & $17(46)$ \\
Male & $20(54)$ \\
\hline Medical specialty & $\mathrm{N}(\%)$ \\
ENT resident & $20(54)$ \\
ENT fellow & $2(5.5)$ \\
ENT surgeon & $15(40.5)$ \\
\hline Medical experience in years & $(\mathrm{QI}$, median, Q3) \\
MD & $3,6,14$ \\
ENT & $0,0,7$ \\
\hline
\end{tabular}

Abbreviations: MD, medicine; ENT, ear, nose and throat.

in the study. Table 1 shows the summary of demographic data of all participants.

A Wilcoxon signed-rank test showed that the satisfaction scores of the in-house manikin were statistically, significantly higher than the satisfaction scores of the commercial manikin for 6 dimensions: proper size, ease of use, ease of maintenance, flexibility of nasal alae relative to actual anatomy, similarity of the nasal cavity relative to actual anatomy and confidence that the manikin can upskill medical students ability to practice on a real patient. On the other hand, the satisfaction scores of the inhouse manikin were not statistically, significantly different from the satisfaction scores of the commercial manikin for 3 dimensions: proper weight, ease of cleaning and well designed for teaching (Table 2).

Simple linear regression showed that none of the variables, including medical specialty, age, gender, time experience as a general physician or an ENT specialist, were independently associated with the total satisfaction score differences between the in-house and commercial manikins (Table 3). As p values, among these independent variables, were all over 0.1 , multiple linear regression was not further analyzed.

\section{Discussion}

As nasal foreign bodies can lead to life-threatening conditions known as foreign body aspiration, adequate training for nasal foreign body removal techniques is required to ensure success in their removal; with minimal attempts in actual patients. Although training in real or simulated patients is not practical and too dangerous, due to a risk of the foreign 
Table 2 A Wilcoxon Signed-Rank Test to Assess the Satisfactions Scores of the In-House and Commercial Manikins

\begin{tabular}{|l|c|c|c|c|}
\hline Dimension & $\begin{array}{c}\text { The In-House Manikin } \\
\text { Median (QI, Q3) }\end{array}$ & $\begin{array}{c}\text { The Commercial Manikin } \\
\text { Median (QI, Q3) }\end{array}$ & z value \\
\hline Proper size & $4(4,5)$ & $4(3,4)$ & -3.840 & $<0.001$ \\
Proper weight & $4(4,5)$ & $4(4,5)$ & -0.832 & -3.882 \\
The ease of use & $5(4,5)$ & $4(3,4)$ & -0.593 & -405 \\
The ease of cleaning & $4(4,5)$ & $4(3,5)$ & -4.158 & $<0.001$ \\
The ease of maintenance & $5(4,5)$ & $3(3,4)$ & -4.092 & $<0.001$ \\
The flexibility of nasal alae & $4(4,5)$ & $3(3,4)$ & -1.209 & $<0.001$ \\
Similarity of the nasal cavity & $4(4,5)$ & $3(3,4)$ & -2.855 & 0.227 \\
A well design for teaching & $4(4,5)$ & $4(4,4)$ & 0.004 \\
Confidence that the manikin can & $4(4,5)$ & $4(3,4)$ & & \\
upskill medical students & & &
\end{tabular}

Table 3 Simple Linear Regression of Variables Associated with the Total Satisfaction Score Difference Between the In-House and Commercial Manikins

\begin{tabular}{|l|c|c|c|c|c|}
\hline Variable & Coefficient & Standard Error & Beta & t & p value \\
\hline Age & 0.664 & 0.668 & 0.176 & 0.994 & 0.328 \\
Gender & 0.157 & 0.111 & 0.246 & 1.413 & 0.168 \\
Medical specialty & -1.099 & 1.787 & -0.11 & -0.615 & 0.543 \\
Medical experience as GP in years & 0.116 & 0.119 & 0.172 & 0.975 & 0.337 \\
Medical experience as ENT in years & 0.106 & 0.126 & 0.15 & 0.847 & 0.403 \\
\hline
\end{tabular}

Abbreviations: GP, general physician; ENT, ear, nose and throat.

body aspiration during the procedure, it has been shown that using a simulated manikin is able to enhance the learning experience of students, and prepare them to practice on real patients with increased knowledge and confidence. ${ }^{13}$

While available commercial manikins for nasal foreign body removal sometimes do not serve all end user's needs, our study showed that an in-house manikin could be devised with high satisfaction scores. The satisfaction scores for the in-house manikin were statistically significantly higher than those of the commercial manikin in a number of dimensions, including proper size, ease of use, ease of maintenance, flexibility of the nasal alae relative to actual anatomy, similarity of the nasal cavity relative to actual anatomy and confidence that the manikin can up-skill the medical students ability to practice on a real patient.

Specifically, the size of the manikin and flexibility of the nasal alae were designed with PU foam and silicone to ensure that it is similar to actual anatomy as much as possible. Furthermore, the particular design of the detachable nose and nasal cavity allowed users to replace these components at any time, if they were torn during training. These factors made the in-house manikin easy to use and for the performance of quick maintenance. However, the limitation of this product was that the head of the manikin was constructed from PU foam. Although PU foam has the benefits of being lighter and is lower in cost than silicone, silicon is more durable than PU foam. Therefore, if durability is of more concern than weight and production cost, future development of a whole manikin could be constructed out of silicone.

It should be noted that the satisfaction scores of some dimensions for the in-house manikin were not statistically significantly different from those with the commercial manikin, for example, to enhance useful feedback, regarding anatomy, and the procedures of the trainers. Nor, did the special design of the detachable nose and nasal cavity of the in-house manikin make the satisfaction scores in the dimension of being well designed for teaching statistically significantly differ from those in the commercial manikin; in which, its nasal cavity section could be also removed from the lateral side. Additionally, the satisfaction scores of both dimensions of proper weight and ease of cleaning for the in-house manikin were not statistically significantly different from those of the commercial manikin. This means the weight of both manikins was not too light, leading to the manikins being displaced further away during the procedure, or not too heavy making the manikins difficult to carry. In addition, both manikins had surfaces which were easy to clean; without grooves or corners 
which can raise cleaning issues. However, based on the simple linear regression to evaluate variables, which might be associated with the satisfaction scores of the manikins, the total satisfaction score difference between both manikins was calculated. We found that no factors, including medical specialty, age, gender, time experience as a general physician or an ENT specialist, could be associated with this difference, that is to say, various specialties, age, gender and time experience did not equate to the in-house manikin having higher scores than those of the commercial manikin.

There were some limitations of this study. First, only a commercial manikin from a single company, which was available in our medical center, was used to compare with the in-house manikin. Obtaining more commercial manikins, from various companies could provide more information regarding advantages and disadvantages between the in-house and other commercial manikins. Second, the participants may have known that the in-house manikins were developed by our institute, so they might have had some bias in evaluation of the satisfaction of each manikin. However, the authors tried to limit this bias by did not influencing the participants prior to the study, and all participants had never seen the in-house model before the study commenced.

\section{Conclusion}

This newly designed in-house nasal foreign body removal manikin had high satisfaction for training, and could be used to develop further nasal foreign body removal manikins in the future.

\section{Data Sharing Statement}

The datasets generated during this current study are available from the corresponding author upon reasonable request.

\section{Ethics Approval and Consent to Participate}

This study was approved by the Ethics Committee and Institutional Review Board of the Faculty of Medicine, Prince of Songkla University. All participants provided informed consent to take part in this study.

\section{Acknowledgments}

We would like to thank Dr Alan Geater and Assistant Professor Thara Tunthanathip, for their useful advice on the statistical analysis. The authors would also like to express their gratitude to Andrew Jonathan Tait, from the International Affairs Department for proofreading this report's English.

\section{Author Contributions}

All authors contributed to data analysis, drafting or revisions of the article, have agreed on the journal to which the article will be submitted. Additionally, the authors have given their final approval for the version to be published, and agree to be accountable for all aspects of the work included.

\section{Funding}

Funding for this research was provided by the Faculty of Medicine, Prince of Songkla University, Songkhla, Thailand. The funders had no role in study design, data collection and analysis, decision to publish nor preparation of the manuscript.

\section{Disclosure}

Dr Pittayapon Pitathawatchai report a patented 2003002767 issued to Kittiphos Anuntaseree; Dr Sittichoke Anuntaseree report a petty patented pending to Application no.2003002767. The authors reported no other conflicts of interest in this work.

\section{References}

1. da Silva BS, Souza LO, Camera MG, Tamiso AG, Castanheira LV. Foreign bodies in otorhinolaryngology: a study of 128 cases. Int Arch Otorhinolaryngol. 2009;13:394-399.

2. Endican S, Garap JP, Dubey SP. Ear, nose and throat foreign bodies in Melanesian children: an analysis of 1037 cases. Int $J$ Pediatr Otorhinolaryngol. 2006;70(9):1539-1545. doi:10.1016/j.ijporl.2006. 03.018

3. Afolabi OA, Suleiman AO, Aremu SK, et al. An audit of paediatric nasal foreign bodies in Ilorin, Nigeria. J Child Health. 2009;3 (2):64-67.

4. Kiger JR, Brenkert TE, Losek JD. Nasal foreign body removal in children. Pediatr Emerg Care. 2008;24(11):785-792. doi:10.1097/ PEC.0b013e31818c2cb9

5. Tong MC, Ying SY, van Hasselt CA. Nasal foreign bodies in children. Int J Pediatr Otorhinolaryngol. 1996;35(3):207-211. doi:10.1016/ 0165-5876(95)01305-9

6. Regonne PE, Ndiaye M, Sy A, Diandy Y, Diop AD, Diallo BK. Nasal foreign bodies in children in a pediatric hospital in Senegal: a three-year assessment. Eur Ann Otorhinolaryngol Head Neck Dis. 2017;134(5):361-364. doi:10.1016/j.anorl.2017.02.013

7. Balatsouras D, Eliopoulos P, Kaberos A, Economou C. Rhinolithiasis: an unusual cause of nasal obstruction. Rhinology. 2002;40:162-164.

8. Awad AH, ElTaher M. ENT Foreign Bodies: an Experience. Int Arch Otorhinolaryngol. 2018;22(02):146-151. doi:10.1055/s-0037-1603922

9. Mackle T, Conlon B. Foreign bodies of the nose and ears in children. Should these be managed in the accident and emergency setting? Int J Pediatr Otorhinolaryngol. 2006;70(3):425-428. doi:10.1016/j. ijporl.2005.07.007 
10. Cohen HA, Goldberg E, Horev Z. Removal of nasal foreign bodies in children. Clin Pediatr (Phila). 1993;32(3):192. doi:10.1177/0009922 89303200319

11. Schulze SL, Kerschner J, Beste D. Pediatric external auditory canal foreign bodies: a review of 698 cases. Otolaryngol Head Neck Surg. 2002;127(1):73-78. doi:10.1067/mhn.2002.126724

12. Halm BM, Lee MT, Franke AA. Improving medical student toxicology knowledge and self-confidence using mannequin simulation. Hawaii Med J. 2010;69:4-7.
13. Swamy M, Sawdon M, Chaytor A, Cox D, Barbaro-Brown J, McLachlan J. A study to investigate the effectiveness of SimMan ${ }^{\circledR}$ as an adjunct in teaching preclinical skills to medical students. BMC Med Educ. 2014;14(1):231. doi:10.1186/14726920-14-231

\section{Publish your work in this journal}

Medical Devices: Evidence and Research is an international, peerreviewed, open access journal that focuses on the evidence, technology, research, and expert opinion supporting the use and application of medical devices in the diagnosis, monitoring, treatment and management of clinical conditions and physiological processes. The identification of novel devices and optimal use of existing devices which will lead to improved clinical outcomes and more effective patient management and safety is a key feature of the journal. The manuscript management system is completely online and includes a very quick and fair peer-review system. Visit http:// www.dovepress.com/testimonials.php to read real quotes from published authors.

Submit your manuscript here: https://www.dovepress.com/medical-devices-evidence-and-research-journal 